\title{
Decreased Tissue Kallikrein Levels and the Risk of Ischemic Stroke: A Community-Based Cross-Sectional Study in China
}

\author{
Xiao Ran' \\ Dao Wen Wang ${ }^{2}$ \\ Zhen $\mathrm{Yu}^{\mathrm{l}}$ \\ Rongxue $\mathrm{Wu}$ (D) $^{3}$ \\ Qin Zhang (1D ${ }^{4}$ \\ 'Department of Emergency, Tongji \\ Hospital, Tongji Medical College, \\ Huazhong University of Science and \\ Technology, Wuhan, 430030, Hubei, \\ People's Republic of China; ${ }^{2}$ The Institute \\ of Hypertension and Department of \\ Internal Medicine, Tongji Hospital, Tongji \\ Medical College, Huazhong University of \\ Science and Technology, Wuhan, 430030, \\ Hubei, People's Republic of China; \\ ${ }^{3}$ Department of Biological Sciences \\ Division/ Cardiology, University of \\ Chicago, Chicago, IL, 60637, USA; \\ ${ }^{4}$ Department of Anesthesiology, Tongji \\ Hospital, Tongji Medical College, \\ Huazhong University of Science and \\ Technology, Wuhan, 430030, Hubei, \\ People's Republic of China
}

Correspondence: Qin Zhang

Email qzhang8@tjh.tjmu.cn

\begin{abstract}
Aim: Tissue kallikrein (TK) exerts protective effects on cardiac cerebrovascular diseases (CCVDs). Changes in TK level in plasma are associated with ischemic stroke and coronary artery disease (CAD); however, a causal correlation could not be established. Therefore, we investigated the association between TK levels and CCVDs in a community-based crosssectional study in China.
\end{abstract}

Methods: A total of 6043 subjects (4242 men and 1801 women) were enrolled in this community-based cross-sectional study. Then, TK levels were measured using an enzymelinked immunosorbent assay kit. Multivariate linear regression model and logistic regression were used to assess the correlations between TK levels and CCVDs. Subsequently, the receiver operating characteristic (ROC) curve was drawn to assess the value of TK level in evaluating the risk of ischemic stroke. Finally, the influence of various medications was evaluated on TK levels.

Results: The TK level was significantly lower in subjects with ischemic stroke $(\mathrm{P}<0.001)$ and hypertension $(\mathrm{P}<0.001)$ and negatively associated with ischemic stroke $(\mathrm{P}<0.001)$ but not associated with hypertension, coronary heart disease, and diabetes compared to the traditional risk factors. The diagnostic accuracy for ischemic stroke, as quantified by the area under the curve, was 0.892 (95\% CI, 0.884-0.900) for TK level, deeming it as a promising assessment tool. Moreover, no appreciable influence of various drugs therapy was found in TK levels ( $\mathrm{P}=0.222)$ except for those taking antilipemic agents.

Conclusion: TK is a strong and independent endogenous protective factor against ischemic stroke in the Chinese population and could be a promising biomarker for the risk of ischemic stroke.

Keywords: tissue kallikrein, ischemic stroke, cardiac cerebral vascular disease, risk factor

\section{Introduction}

Cardiovascular and cerebrovascular diseases (CCVDs) are the main chronic noncommunicable diseases that endanger human health. Stroke and coronary artery disease $(\mathrm{CAD})$ are the leading causes of death and the primary cause of permanent disability in adults globally. ${ }^{1-3}$ In the 20 years from 2010 to 2029, as China's population grows and ages, the number of patients with stroke and coronary heart disease will increase significantly. The prevalence of stroke among people $>25$ years-old is leading in China. Ischemic stroke is the primary type, accounting for up to $80 \%$ of the incidence of stroke. ${ }^{1,4}$ Experimental data suggested that tissue kallikrein (TK), a serine protease, cleaves low molecular kininogen to release 
bradykinin, which then exerts a series of cardiocerebrovascular protective effects through bradykinin B2 receptor (B2R).$^{5-7}$ CCVDs and their risk factors, such as hypertension, diabetes, $\mathrm{CAD}$, and ischemic stroke, are associated with TK. ${ }^{1,8-10}$ Thus, we hypothesized that changes in TK levels might be a risk factor for CCVD.

The results of our previous study demonstrated that a decrease in TK level was associated with the incidence and recurrence of ischemic stroke, predicting a high risk of stroke recurrence. ${ }^{11}$ This finding was consistent with basic research, suggesting the protective effect of TK on ischemic stroke. ${ }^{12,13}$ However, the level of TK was higher in CAD patients in another study ${ }^{14}$ and is implicated in protecting against CAD. Thus, the association of TK levels with ischemic stroke and CAD patients is contradictory, although both are atherosclerotic diseases. Therefore, it could be speculated that decreased TK level is a specific biomarker of stroke.

Based on existing clinical studies, ${ }^{11,14}$ TK levels were assessed in various case-control studies, but a causal link between reduced plasma levels of TK and the risk of CCVDs could not be established. Moreover, the influence of medicine on plasma TK levels cannot be ignored. Therefore, we set up a multicenter community-based cross-sectional study to investigate the correlation between TK levels and CCVDs and their risk factors and to evaluate the association of TK level with ischemic stroke, $\mathrm{CAD}$, hypertension, and diabetes. Also, the effect of medical treatment on TK level was assessed in this study.

\section{Methods}

\section{Study Design, Setting, and Population}

The primary community-based cross-sectional study population has been described previously. ${ }^{15}$ Herein, we recruited participants aged 20-80 years from January 17, 2009 to August 7, 2010, in Rizhao City in the northern region of China. A multistage cluster sampling method was applied. Each participant was interviewed and asked to complete a standardized questionnaire consisting of a range of demographic factors, medical history, history of medications, and lifestyle. The traditional risk factors of CCVDs, such as age, sex, weight, body mass index (BMI), diastolic blood pressure (DBP), systolic blood pressure (SBP), alcohol consumption, current smoking, status of hypertension, diabetes, hyperlipidemia, and stroke, were recorded in all the subjects. Among these, the status of regular drug treatment (angiotensin-converting enzyme inhibitors (ACEIs)/angiotensin receptor blockers (ARBs), calcium channel blockers, beta blockers, diuretics, antilipemic agents, hypoglycemic drugs, NSAIDs and other drugs) were recorded as well. Stroke patients were diagnosed by rigorous neurological examination, including computed tomography, magnetic resonance imaging, or both. Imaging data were reviewed by experienced neuroradiologists to confirm the diagnosis and stroke subtypes. ${ }^{16}$ CAD was defined by one or more of the following diagnostic criteria: 1) patients who were documented by coronary angiography to have at least a $50 \%$ stenosis in a major epicardial artery and 2) patients with a history of coronary artery bypass graft or percutaneous coronary intervention. ${ }^{17}$ Hypertension was defined as DBP $\geq 90$ $\mathrm{mmHg}$ and/or SBP $\geq 140 \mathrm{mmHg}$, physician diagnosis, or current medication for hypertension. ${ }^{18}$ Diabetes was defined according to the criteria of the American Diabetes Association. ${ }^{19}$ Hyperlipidemia was defined as TC $>6.2 \mathrm{mmol} / \mathrm{L}$ and $/$ or $\mathrm{TG}>2.3 \mathrm{mmol} / \mathrm{L}^{20}$ Initially, 6487 participants from the community population were enrolled. However, before data evaluation, 444 subjects were excluded due to missing samples and data. Finally, a total of 6043 subjects completed the survey, yielding a response rate of $93.16 \%$.

The study was governed under the 2007-2008 version of the World Medical Association's Declaration of Helsinki. The study protocol was approved by the review board of the Ministry of Public Health and the Ministry of Science and Technology of China, and the ethics committees of the Fuwai Hospital and Tongji Hospital Affiliated to Tongji Medical College. Participation was voluntary; informed consent was obtained from each participant. All investigators were trained at the Cardiovascular Institute, Chinese Academy of Medical Sciences (Beijing, China), and qualified for the clinical investigation.

\section{Plasma TK Assays}

Blood specimens were obtained from an antecubital vein after overnight fasting. Plasma was separated and stored at $-80^{\circ} \mathrm{C}$ until further analysis. From May 30, 2013 to June 20, 2013, TK levels were measured in 6043 plasma samples using double antibody sandwich biotin-avidin enzymelinked immunosorbent assay kit which are prepared by ourselves and described in our previous study. ${ }^{11}$ The microplates were pre-coated with anti-TK polyclonal antibody and incubated at $4{ }^{\circ} \mathrm{C}$ for $24 \mathrm{~h}$. After aspirating each well and washing, the plates were blocked with $5 \%$ bovine serum albumin. A volume of $100 \mu \mathrm{L}$ of the standards and samples was added 
to each well and incubated at $37^{\circ} \mathrm{C}$ for $1 \mathrm{~h}$. Subsequently, a biotin-conjugated monoclonal antibody prepared specifically for TK was added to the appropriate microplate wells for $40 \mathrm{~min}$ along with avidin conjugated to horseradish peroxidase. Then, a 3,3',5,5'-tetramethylbenzidine (TMB) substrate solution was added to each well. Finally, the enzyme-substrate reaction was terminated, and the absorbance was determined at $450 \mathrm{~nm}$.

\section{Statistical Analysis}

Demographics and clinical and laboratory variables were described as mean \pm standard deviation (SD) and as frequency for categorical variables. Continuous variables were expressed as mean \pm standard deviation, and independent-samples $t$-test or one-way analysis of variance (ANOVA) was used to indicate statistical significance. The categorical variables were analyzed by chi-square test. Pearson's and Spearman correlation coefficients were used to test the correlation between continuous and categorical variables. Receiver operating characteristic (ROC) curves were plotted to evaluate the TK level while assessing the risk of ischemic stroke.

Since the distribution of plasma TK levels was skewed (identified using assessment of skewness and kurtosis), all the subjects were grouped by quartiles according to plasma TK levels, which were then used as continuous variables and categorical variables in the following analysis. Unconditional logistic regression analysis was used to calculate the odds ratios (ORs) and their 95\% confidence intervals (CIs) after adjustment for potential confounders. A two-tailed value of $\mathrm{P}<0.05$ was considered significant.

We used independent-samples $t$-test and ANOVA to compare the TK levels for each medication (present/ absent) and performed logistic regression analysis to evaluate the TK levels on the risk of ischemic stroke with respect to traditional risk factors and the status of drug therapy. SPSS 18.0 (IBM, Armonk, NY, USA) and MedCalc 15.8 (MedCalc Software Ltd, Ostend, Belgium) software were used for statistical analyses.

\section{Results}

\section{Baseline Characteristics of the Population}

A total of 6043 subjects (4242 men and 1801 women) were analyzed in this community-based cross-sectional study. The demographic and clinical characteristics of the population are summarized in Table 1. Among them, 70\% were males with a mean age of $43 \pm 12$ years, bodyweight of $72 \pm 12 \mathrm{~kg}$, BMI of $25 \pm 5 \mathrm{~kg} / \mathrm{m}^{2}, \mathrm{SBP}$ of $121 \pm 17 \mathrm{mmHg}$,

Table I Demographic and Clinical Characteristics of Study Population*

\begin{tabular}{|c|c|c|c|c|c|c|}
\hline Characteristics & Overall & Quartile I & Quartile 2 & Quartile 3 & Quartile 4 & $\mathbf{p}^{\dagger}$ \\
\hline TK levels, mg/l & $0.304 \pm 0.115$ & $<0.260$ & $0.260-0.282$ & $0.282-0.359$ & $>0.359$ & \\
\hline No. of participants & 6043 & 1510 & 1511 & $151 \mid$ & 1511 & \\
\hline Male sex, $\mathrm{n}(\%)$ & $4242(70)$ & $1134(75)$ & $1107(73)$ & $1030(68)$ & $971(64)$ & 0.001 \\
\hline Hypertension, n(\%) & $992(16)$ & $285(19)$ & $23 I(15)$ & $246(16)$ & $227(15)$ & 0.782 \\
\hline Ischemic stroke, $n(\%)$ & $76(1.3)$ & $66(4.4)$ & $\mathrm{I}(0 . \mathrm{I})$ & $8(0.5)$ & $\mathrm{I}(0 . \mathrm{I})$ & $<0.001$ \\
\hline CAD, n(\%) & $255(4.2)$ & $66(4.4)$ & $\mathrm{I}(3.7)$ & $70(4.6)$ & $63(4.2)$ & 0.954 \\
\hline Diabetes, $\mathrm{n}(\%)$ & $223(3.7)$ & $64(4.2)$ & $54(3.6)$ & $53(3.5)$ & $52(3.4)$ & 0.297 \\
\hline Hyperlipidemia, n(\%) & $1214(20)$ & $4 \mid 2(27)$ & $273(18)$ & $275(18)$ & $254(17)$ & $<0.001$ \\
\hline Alcohol consumption, n (\%) & $2704(45)$ & $753(49)$ & $711(47)$ & $623(4 I)$ & $617(4 I)$ & 0.11 \\
\hline Current smoker, n(\%) & $2190(36)$ & $555(37)$ & $526(35)$ & $536(35)$ & $573(38)$ & $<0.001$ \\
\hline Family history of hypertension, $\mathrm{n}(\%)$ & $2165(36)$ & $554(37)$ & $526(35)$ & $536(36)$ & $549(36)$ & $<0.001$ \\
\hline Family history of stroke, $n(\%)$ & $437(7.2)$ & $126(8.3)$ & $94(6.2)$ & $102(6.8)$ & $115(7.6)$ & 0.646 \\
\hline Family history of CAD, $n(\%)$ & $708(12)$ & $159(11)$ & $184(12)$ & $186(12)$ & $179(12)$ & 0.393 \\
\hline Age, y & $43 \pm 12$ & $42 \pm 12$ & $42 \pm 12$ & $44 \pm 12$ & $44 \pm 12$ & $<0.001$ \\
\hline Waist circumference & $86 \pm 10$ & $87 \pm 10$ & $86 \pm 10$ & $85 \pm 10$ & $85 \pm 10$ & 0.068 \\
\hline Abdomen circumference & $99 \pm 6$ & $99 \pm 6$ & $99 \pm 6$ & $99 \pm 6$ & $99 \pm 6$ & 0.074 \\
\hline BMI, $\mathrm{kg} / \mathrm{m}^{2}$ & $25 \pm 5$ & $26 \pm 6$ & $26 \pm 7$ & $25 \pm 3$ & $25 \pm 3$ & 0.005 \\
\hline Weight, kg & $72 \pm 12$ & $72 \pm 12$ & $72 \pm 12$ & $71 \pm 12$ & $71 \pm 12$ & 0.058 \\
\hline SBP, mm Hg & $121 \pm 17$ & $122 \pm 17$ & $120 \pm 17$ & $121 \pm 18$ & $120 \pm 17$ & 0.939 \\
\hline DBP, $\mathrm{mm} \mathrm{Hg}$ & $80 \pm 11$ & $8 I \pm 11$ & $80 \pm 11$ & $80 \pm 11$ & $79 \pm 11$ & 0.055 \\
\hline
\end{tabular}

Notes: *All the subjects (4242 men and I80I women) were grouped by quartiles according to plasma tissue kallikrein (TK) levels: Quartile I, Quartile 2, Quartile 3 and Quartile 4. ${ }^{\dagger} \mathrm{p}$-values for the correlations between TK levels and characteristics in multivariate linear regression model.

Abbreviations: TK, tissue kallikrein; CAD, coronary artery disease; BMI, body mass index; SBP, systolic blood pressure; DBP, diastolic blood pressure. 


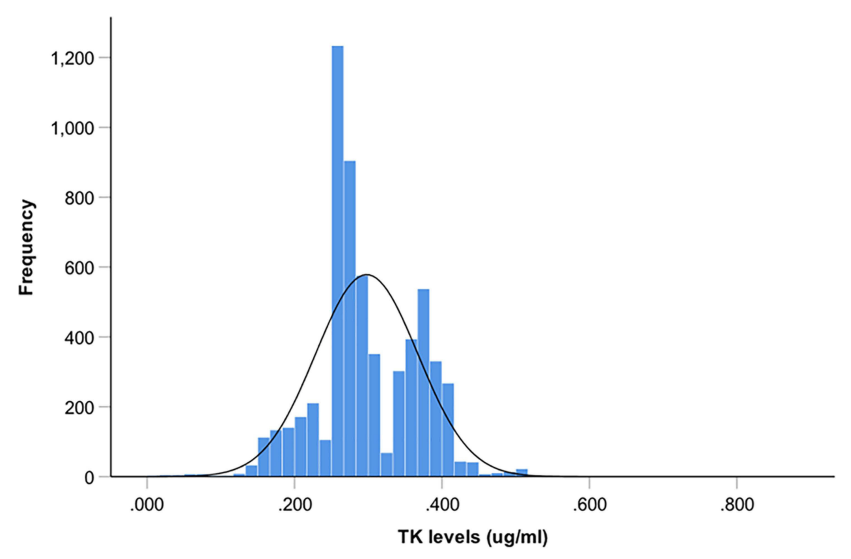

Figure I Distribution of plasma TK levels. Distribution of plasma TK levels subjected to positively skewed distribution $(0.304 \pm 0.115 \mathrm{mg} / \mathrm{mL}$; skewedness = 3.187 , standard error $=0.032$; kurtosis $=59.417$, standard error $=0.063$ ).

DBP of $80 \pm 11 \mathrm{mmHg}$, the waist circumference of 86 $\pm 10 \mathrm{~cm}$, and abdomen circumference of $99 \pm 6 \mathrm{~cm}$. Moreover, $16 \%$ of the participants had hypertension, $1.3 \%$ had an ischemic stroke, $4.2 \%$ had CAD, 3.7\% had diabetes, and $20 \%$ had hyperlipidemia in this study, while the prevalence of alcohol consumption and smoking was $45 \%$ and $36 \%$, respectively.

To evaluate the correlation between TK levels and CCVDs, the plasma TK levels of all the subjects were measured, and the results showed that the distribution of TK was positively skewed $(0.304 \pm 0.115 \mathrm{mg} / \mathrm{mL}$; skewedness $=3.187$, standard error $=0.032$; kurtosis $=59.417$, standard error $=0.063$; Figure 1). According to the levels of $\mathrm{TK}$, all subjects were grouped in quartiles (quartile 1, $<0.260 \mathrm{mg} / \mathrm{mL}$; quartile $2,0.260-0.282 \mathrm{mg} / \mathrm{mL}$; quartile 3, 0.282-0.359 $\mathrm{mg} / \mathrm{mL}$; quartile $4,>0.359 \mathrm{mg} / \mathrm{mL}$ ) for further analysis (Table 1).

\section{Association of TK Levels with CCVDs}

The correlations between TK levels and CCVDs and their risk factors are presented in Table 1. In the multivariate linear regression model, TK levels were positively associated with gender $(t=9.040 ; \mathrm{P}<0.001)$ and smoking $(t=6.215 ; \mathrm{P}<0.001)$ and negatively associated with male sex $(t=-3.387 ; \quad \mathrm{P}=0.001), \quad$ ischemic stroke $(\mathrm{t}=-9.950$; $\mathrm{P}<0.001)$, hyperlipidemia $(t=-5.955 ; \mathrm{P}<0.001)$, BMI $(t=$ $-2.830 ; \mathrm{P}=0.005)$, and family history of hypertension $(t=$ $-6.109 ; \mathrm{P}<0.001)$.

Data did not reveal any significant difference in TK levels between subjects with CAD $(0.299 \pm 0.074$ vs 0.298 $\pm 0.069 \mathrm{mg} / \mathrm{mL}, \mathrm{P}=0.753$; Figure $2 \mathrm{~A})$ and diabetes $(0.293$ \pm 0.069 vs $0.298 \pm 0.069 \mathrm{mg} / \mathrm{mL}, \mathrm{P}=0.262$; Figure $2 \mathrm{~B}$ ) and the controls. As described in Table 2, TK level was not associated with the risk of $\mathrm{CAD}(\mathrm{OR}=1.009,95 \% \mathrm{CI}$ : 0.886-1.148, $\mathrm{P}=0.893$; Figure $2 \mathrm{~F})$ ) and diabetes $(\mathrm{OR}=0.935,95 \% \mathrm{CI}: 0.822-1.063, \mathrm{P}=0.304$; Figure $2 \mathrm{~F}$ ). The TK level was significantly lower in subjects with hypertension than in those without hypertension $(0.291$ \pm 0.073 vs $0.299 \pm 0.069 \mathrm{mg} / \mathrm{mL}, \mathrm{P}<0.001$; Figure $2 \mathrm{C}$ ) but was much higher than that in ischemic stroke patients $(0.291 \pm 0.067 \quad$ vs $\quad 0.155 \pm 0.094 \quad \mathrm{mg} / \mathrm{mL}, \quad \mathrm{P}<0.0001$; Figure 2D) and thus, could not be a risk factor of hypertension $\quad(\mathrm{OR}=0.969,95 \% \quad \mathrm{CI}: 0.893-1.050, \quad \mathrm{P}=0.438$; Table 2 and Figure 2F). However, we found that TK levels were lower in subjects with ischemic stroke $(0.155 \pm 0.094$ vs $0.300 \pm 0.067 \mathrm{mg} / \mathrm{mL}, \mathrm{P}<0.0001$; Figure $2 \mathrm{E}$ ) and negatively associated with the risk of ischemic stroke adjusting the traditional risk factors $(\mathrm{OR}=0.198,95 \% \mathrm{CI}: 0.131-$ $0.298, \mathrm{P}<0.001$; Table 2 and Figure $2 \mathrm{~F}$ ). This phenomenon suggested that TK level is an independent risk factor of ischemic stroke.

\section{TK Levels on the Risk of Ischemic Stroke}

Next, the ROC curve was plotted, which showed that the evaluation value of plasma TK level on the risk of ischemic stroke was reliable (area under the curve (AUC) $(95 \%$ CI $)=0.892(0.884-0.900), \quad Z=15.821$, $\mathrm{P}<0.001$; Figure 3). A plasma TK level of $0.235 \mathrm{mg} / \mathrm{mL}$ had a sensitivity of $87 \%$, specificity of $87 \%$, and Youden index of 0.741 . Supposedly, plasma TK levels could be a promising biomarker for the risk of ischemic stroke.

\section{Influence of Drug Therapy on TK Level}

TK lowers the blood pressure, ${ }^{21-23}$ and hence, we suspected that antihypertensive agents or other medicines might influence the level of TK in hypertensive subjects. To evaluate the influence of various drug treatments on the TK level, we recorded the status of the therapy in all the participants. Untreated population was younger, lighter weight and had lower BMI, waist circumference, abdomen circumference and blood pressure than those with regular drug therapy (Table 3). Also, they were less likely to have a history of hypertension, stroke, CAD, diabetes, hyperlipidemia, and smoking and a family history of hypertension, stroke and CAD (Table 3). However, the TK level did not differ between participants with the presence and absence of drug therapy $(0.296 \pm 0.074$ vs $0.298 \pm 0.069 \mathrm{mg} /$ $\mathrm{mL}, \mathrm{P}=0.407$; Figure 4A). Further analysis did not find distinct differences in the TK level among subjects with ACEIs/ARBs, calcium channel blockers, beta-blockers, 
A

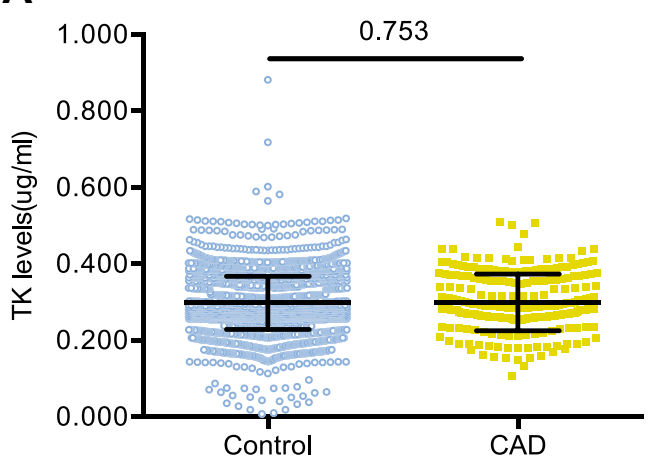

C

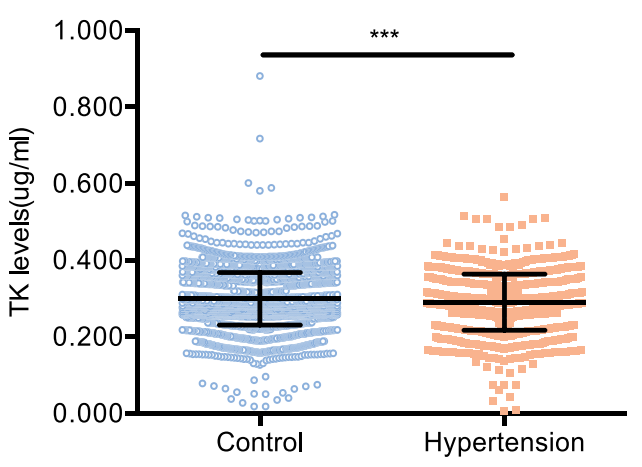

E

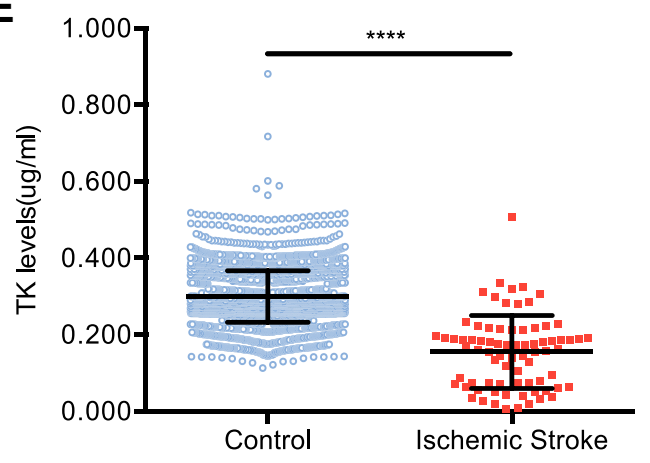

B

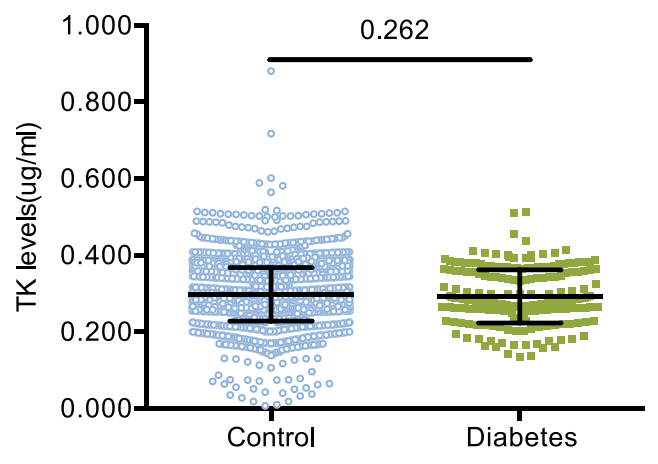

D

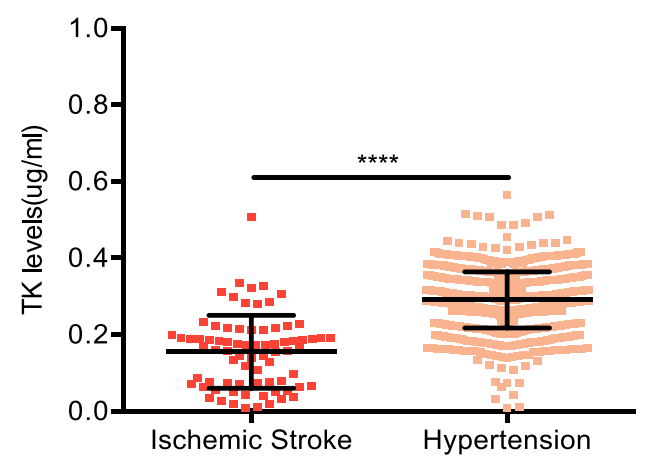

$\mathbf{F}$

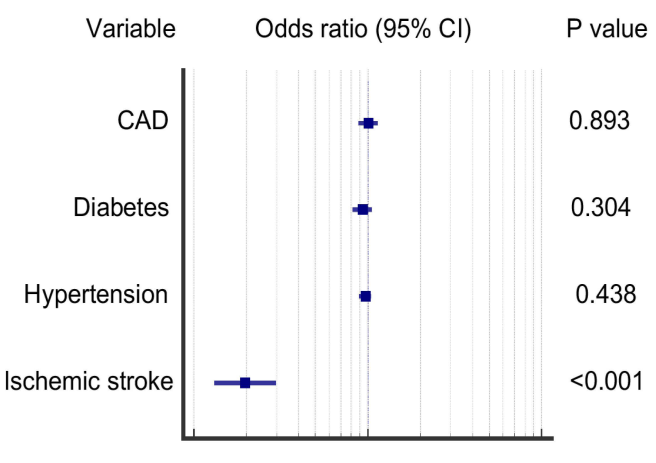

Figure 2 Association of TK levels with CAD, diabetes, hypertension and ischemic stroke. (A) Mean plasma TK level in subjects grouped by the status of CAD. (B) Mean plasma TK level in subjects grouped by the status of diabetes. (C) Mean plasma TK level in subjects grouped by the status of hypertension; ***** $<0.00 \mathrm{I}$. (D) Mean plasma TK level in subjects grouped by the status of ischemic stroke; $* * * * \mathrm{P}<0.00 \mathrm{I}$. (E) Mean plasma TK level in hypertensives and ischemic stroke patients; $* * * * \mathrm{P}<0.00 \mathrm{I}$. (F) Forest plot showing adjusted logistic regression analysis. Odds ratio (black dots) and the corresponding confidence intervals (black lines) for the risk of TK levels in CAD, diabetes, hypertension and ischemic stroke.

diuretics, hypoglycemic drug, nonsteroidal antiinflammatory drugs (NSAIDs), other drugs, and those without medicine $(0.299 \pm 0.072$ vs $0.290 \pm 0.071$ vs 0.289 \pm 0.074 vs $0.296 \pm 0.072$ vs $0.293 \pm 0.070$ vs $0.292 \pm 0.072$ vs $0.304 \pm 0.072 \quad$ vs $\quad 0.298 \pm 0.069 \quad \mathrm{mg} / \mathrm{mL}, \quad \mathrm{P}=0.222$; Figure 4B). However, subjects taking antilipemic agents had lower TK levels than the untreated group (0.271 \pm 0.069 vs $0.298 \pm 0.069 \mathrm{mg} / \mathrm{mL}, \mathrm{P}<0.001$; Figure 4B). Considering the traditional risk factors and the status of drug therapy, plasma TK level was still negatively associated with the risk of ischemic stroke ( $\mathrm{OR}=0.197$, 95\% CI: 0.131-0.297, $\mathrm{P}<0.001$ ), suggesting no appreciable influence of various medications on TK levels in this study.

\section{Discussion}

To the best of our knowledge, the present study systematically revealed the correlation between TK levels and CCVDs and their traditional risk factors in a community-based crosssectional study for the first time. We found that the levels of 
Table 2 TK Levels and the Risk of Coronary Artery Disease (CAD), Diabetes, Hypertension and Ischemic Stroke

\begin{tabular}{|l|c|c|c|c|c|}
\hline Groups and Measure & No. of Participants & Unadjusted* & p & Adjusted* & p \\
\hline CAD & 255 & $1.007(0.900-1.126)$ & 0.908 & $1.009(0.886-1.148)$ & 0.893 \\
Diabetes & 223 & $0.932(0.826-1.050)$ & 0.246 & $0.935(0.822-1.063)$ & 0.304 \\
Hypertension & 992 & $0.938(0.866-1.015)$ & 0.112 & $0.969(0.893-1.050)$ & 0.438 \\
Ischemic stroke & 76 & $0.209(0.139-0.313)$ & $<0.001$ & $0.198(0.131-0.298)$ & $<0.001$ \\
\hline
\end{tabular}

Notes: *Odds ratio (95\% confidence interval). Adjusted odds ratios have been adjusted for age, sex, weight, body mass index, systolic blood pressure, diastolic blood pressure, presence or absence of CAD, diabetes, hypertension, ischemic stroke, current smoking and alcohol consumption, family history of hypertension, CAD and stroke.

TK were lower in subjects with ischemic stroke and slightly lower in hypertensive patients, but no change was detected in the participants with CAD and diabetes. Notably, considering the traditional risk factors of CCVDs, TK level was shown to be negatively associated with the risk of ischemic stroke but not correlated to hypertension, $\mathrm{CAD}$, and diabetes, suggesting that decreased TK level is an independent and specified risk factor of ischemic stroke. Furthermore, the ROC curve also proved a strong evaluation value of TK level on the risk of ischemic stroke. Our previous study found that TK levels were lower in ischemic stroke patients than in healthy controls and predicted higher recurrence rates and shorter survival times. ${ }^{11}$ However, the case-control study can neither demonstrate a causal correlation between reduced TK levels and stroke nor can reveal whether TK levels could be used as biomarkers for the diagnosis of stroke. As a cross-sectional study in a community population, the present study demonstrated the correlation between TK levels and ischemic stroke and

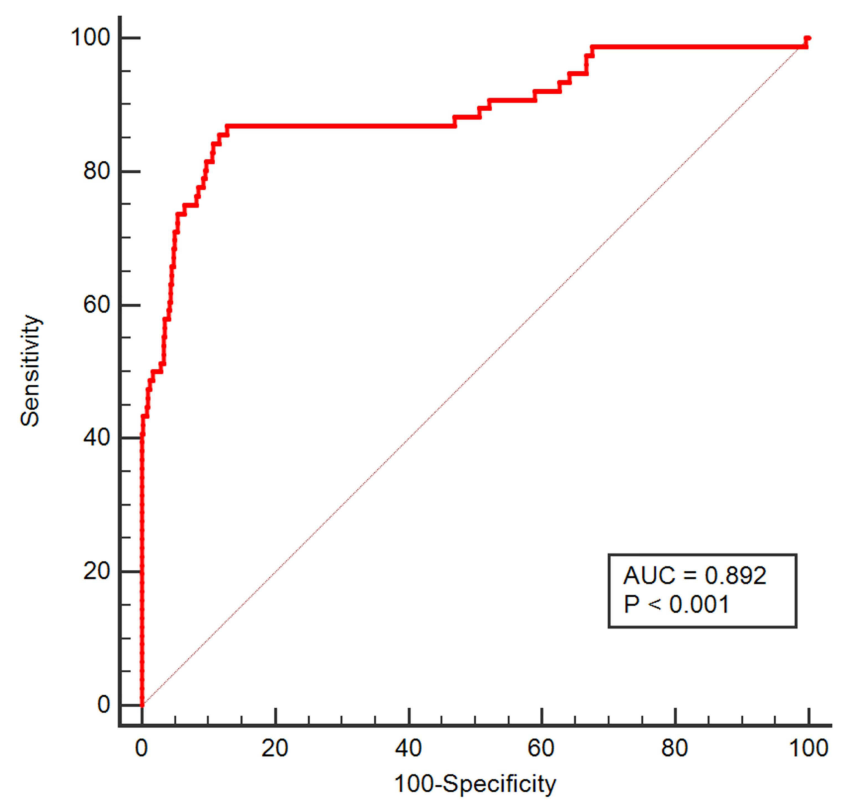

Figure 3 ROC curve for diagnosis of ischemic stroke using TK level. Note: AUC, reflecting high diagnostic value. suggested that reduced TK levels may be used as a risk factor of ischemic stroke. Taken together, TK is a strong and independent endogenous protective factor against ischemic stroke in the Chinese population and could be a promising biomarker for the risk of ischemic stroke.

TK is an endogenous protective factor of hypertension, although it is not associated with the risk of hypertension in the present study. Early basic studies demonstrated that TK, a serine protease processes low-molecular-weight kininogen substrates to release vasoactive bradykinin and kinin and then lowers the blood pressure by activating endothelial NO synthase (eNOS) and increasing NO production., 5,7,24 Antihypertensive agents, such as ACEIs and ARBs lower the blood pressure and other cardiac-cerebral vascular protection by inhibiting the activity of the renin-angiotensin system (RAS) ${ }^{25}$ Cardiovascular homeostasis is derived from a balance between RAS and the kallikrein-kinin system (KKS). The double-action of ACEIs is blocked by RAS and the potentiation of the KKS. ${ }^{26}$ Also, TK is a key enzyme in $\mathrm{KKS}^{6}$ that triggers a cascade of biological effects, and some antihypertensive agents might affect the levels of TK. Thus, we considered the status of medicine administration into account in the present study. These findings were consistent with those from our previous study ${ }^{11}$ indicating that various medicines did not affect the TK levels and would not change the results of this study. This could be attributed to the fact that ACEIs inhibit KKS by reducing the degradation of bradykinin instead of $\mathrm{TK},{ }^{27}$ and therefore may not affect the level of TK.

KKS reduces the elevated blood pressure, ${ }^{28}$ has an antiinflammatory effect, ${ }^{29}$ protects against ischemia/reperfusion damage, ${ }^{30}$ and inhibits the proliferation of vascular smooth muscle cells and neointima formation in arteries. ${ }^{31}$ Consequently, it attenuates arteriosclerosis and atherosclerosis ${ }^{26}$ and prevents stroke and CAD. Interestingly, plasma TK level was lower in stroke patients and higher in CAD patients in our previous study, although numerous studies have demonstrated the independent cardio-cerebrovascular protective effect of TK in animal models. TK also has 
Table 3 Baseline Characteristics of Subjects Grouped by the Status of Drug Therapy

\begin{tabular}{|c|c|c|c|}
\hline Characteristics & Regular Drug Therapy & No Treatment & $\mathbf{p}^{\dagger}$ \\
\hline No. of participants & 693 & 5350 & \\
\hline Male sex, n (\%) & 489 (7I) & $3753(70)$ & 0.823 \\
\hline Hypertension, n (\%) & $601(87)$ & $388(7)$ & $<0.001$ \\
\hline Stroke, n (\%) & $29(4)$ & $47(\mathrm{I})$ & $<0.001$ \\
\hline CAD, n (\%) & $|4|(20)$ & $114(2)$ & $<0.001$ \\
\hline Diabetes, n (\%) & $97(14)$ & $126(2)$ & $<0.001$ \\
\hline Hyperlipidemia, n (\%) & $303(44)$ & $911(17)$ & $<0.001$ \\
\hline Alcohol consumption, $\mathrm{n}(\%)$ & $325(47)$ & $2379(44)$ & 0.226 \\
\hline Current smoker, n (\%) & $359(52)$ & $|83|(34)$ & $<0.001$ \\
\hline Family history of hypertension, $\mathrm{n}(\%)$ & $355(5 \mathrm{I})$ & $1810(34)$ & $<0.001$ \\
\hline Family history of stroke, $\mathrm{n}(\%)$ & $96(14)$ & $341(6)$ & $<0.001$ \\
\hline Family history of CAD, n (\%) & $117(17)$ & $591(\mathrm{II})$ & $<0.001$ \\
\hline Age, y & $56 \pm 11$ & $4 I \pm I I$ & $<0.001$ \\
\hline Waist circumference & $92 \pm 9$ & $85 \pm 10$ & $<0.001$ \\
\hline Abdomen circumference & $102 \pm 6$ & $101 \pm 6$ & $<0.001$ \\
\hline $\mathrm{BMI}, \mathrm{kg} / \mathrm{m}^{2}$ & $27 \pm 7$ & $25 \pm 5$ & $<0.001$ \\
\hline Weight, kg & $74 \pm 11$ & $7 I \pm 12$ & $<0.001$ \\
\hline SBP, mm Hg & $138 \pm 18$ & $118 \pm 16$ & $<0.001$ \\
\hline DBP, mm Hg & $87 \pm 12$ & $79 \pm 11$ & $<0.001$ \\
\hline TK levels, mg/l & $0.296 \pm 0.074$ & $0.298 \pm 0.069$ & 0.407 \\
\hline
\end{tabular}

Note: ${ }^{\dagger} \mathrm{P}$-values for the difference between subjects with regular drug therapy and untreatment.

Abbreviations: TK, tissue kallikrein; CAD, coronary artery disease; BMI, body mass index; SBP, systolic blood pressure; DBP, diastolic blood pressure.

a strong hypotensive effect. ${ }^{8}$ As expected, the TK level was lower in hypertensive patients but did not show any significant association with the risk of hypertension. To address whether decreased TK level is a specific biomarker of ischemic stroke, we evaluated the correlation between TK levels and CCVDs and their risk factors in this community-based cross-sectional study and found that TK level was significantly negatively associated with the risk of ischemic stroke but not correlated with $\mathrm{CAD}$, hypertension, and diabetes, suggesting that TK might be a specific biomarker of ischemic stroke. Current

\section{A}

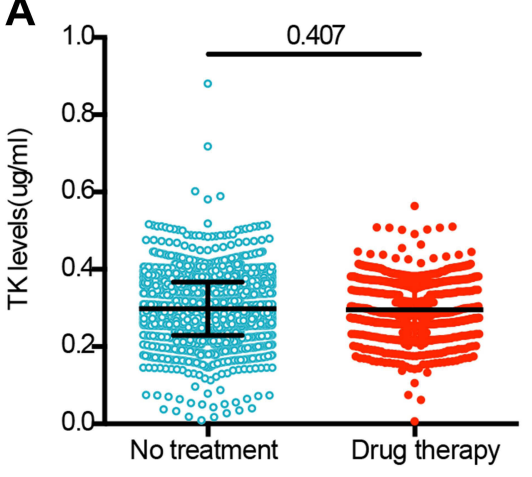

B

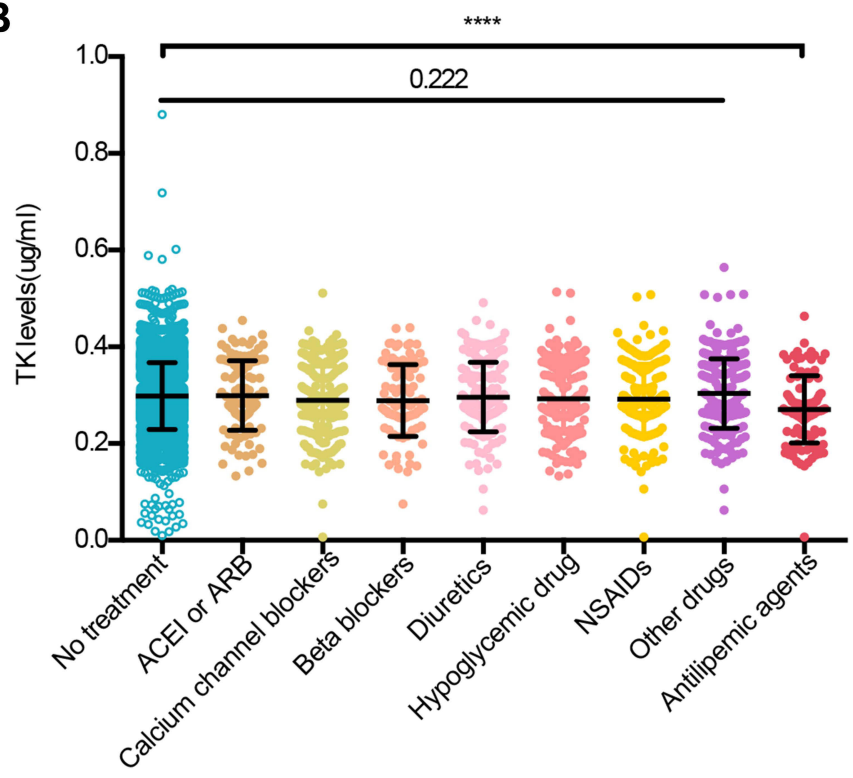

Figure 4 Mean plasma TK level in subjects grouped by the status of regular drug therapy. (A) Plasma TK level in subjects with regular drug therapy and no treatment. (B) Plasma TK level in subjects grouped by the administration of various medicines. ${ }^{*} * * * \mathrm{P}<0.001$. 
studies proposed that the protective effect of TK on cardiovascular and cerebrovascular systems is exerted via B2R/eNOS signaling pathway. ${ }^{6,13,32} \mathrm{~B} 2 \mathrm{R}$ is widely distributed in the vascular endothelial cells of different organs ${ }^{26}$ but barely expressed in normal brain microvascular endothelial cells. ${ }^{33}$ These phenomena may partially explain why the decreased plasma TK level is a specific biomarker of the ischemic stroke instead of other CCVDs, although the exact reasons and mechanisms are yet to be explored. Collectively, TK is a strong endogenous protective factor against CCVDs and an independent and specific biomarker of ischemic stroke.

Dyslipidemia is a well-known causal risk factor of the development of atherosclerosis, which is the pathological basis of most cardiovascular diseases such as CAD and ischemic stroke, and the leading cause of morbidity and mortality worldwide. ${ }^{34,35}$ In addition, TK inhibits glycogen accumulation and improves glucose utilization and lipid metabolism via activation of the Akt/GSK-3 $\beta$ signaling pathway. ${ }^{36}$ Consistent with our previous studies in stroke ${ }^{11}$ and $\mathrm{CAD},{ }^{14} \mathrm{TK}$ was negatively correlated with hyperlipidemia in the present study. Moreover, we found that hypertension patients taking antilipemic agents had lower TK levels than untreated subjects, suggesting that the TK level might be regulated by lipid metabolism. Therefore, reduced TK levels may increase the risk of ischemic stroke by downregulating the B2R/eNOS/Akt/GSK-3 $\beta$ signaling pathway leading to dyslipidemia and promoting atherosclerosis. Human urinary kallidinogenase (HUK) is tissue kallikrein (TK) extracted from human urine and used in the treatment of ischemic stroke in China. ${ }^{37}$ Exogenous HUK increases the cerebral blood flow, ameliorates neurological deficits, and then improves the outcomes in patients with ischemic stroke, ${ }^{38,39}$ suggesting that regulating the TK level might have specific implications in the treatment of stroke.

Nevertheless, the primary limitation of this retrospective survey is that it is a community-based cross-sectional study without follow-up data. TK levels were assessed after collecting the clinical characteristics of the population; therefore, we could not establish a causal link between reduced plasma levels of TK and ischemic stroke or determine whether changes in the TK levels are predictors or consequences of CVVDs. However, we evaluated the changes in TK level and the risk of CCVDs in this multicenter community-based cross-sectional study and suggested that reduced TK level may be a specific risk factor of ischemic stroke. Furthermore, the ROC curve proved the assessed value of reduced TK level on the risk of ischemic stroke. Therefore, additional prospective studies with follow-up data would establish a causal link between reduced plasma levels of TK and ischemic stroke in the future. In addition, we adjusted for many risk factors in the multivariate analysis, but residual confounding factors could not be excluded. Second, it is difficult to ignore the influence of the administered medicines on the plasma TK levels at all, although the influences of various drugs were not apparent in this study. However, hypertensive patients taking antilipemic agents had lower TK levels than untreated subjects. These issues could be addressed by a cohort study of changes in the TK levels before and after taking the medicine. Moreover, basic studies would need to elucidate why reduced TK levels are specific risk factors for ischemic stroke in the future.

\section{Conclusions}

Overall, the present study suggested that decreased TK level may be an independent, specific risk factor for ischemic stroke, and regulating the TK level may have important implications for the treatment of stroke.

\section{Abbreviations}

TK, tissue kallikrein; CCVDs, cardiac cerebral vascular diseases; $\mathrm{CAD}$, coronary artery disease; $\mathrm{B} 2 \mathrm{R}$, bradykinin B2 receptor; BMI, body mass index; DBP, diastolic blood pressure; SBP, systolic blood pressure; TMB, 3,3',5,5'tetramethylbenzidine; ANOVA, one-way analysis of variance; ROC, receiver operating characteristic; OR, odds ratio; CI, confidence interval; KKS, kallikrein-kinin system; HUK, human urinary kallidinogenase.

\section{Data Sharing Statement}

The data, analytical methods, and study materials will be made available from the corresponding author upon reasonable request after institutional approval and according to the institutional process.

\section{Acknowledgments}

We thank Dr Rutai Hui for his help in obtaining plasma samples and all the investigators, clinical research coordinators and data managers involved in the study for their dedicated contributions.

\section{Author Contributions}

All authors made a significant contribution to the work reported, whether that is in the conception, study design, execution, acquisition of data, analysis and interpretation, or in all these areas; took part in drafting, revising or critically reviewing the article; gave final approval of the 
version to be published; have agreed on the journal to which the article has been submitted; and agree to be accountable for all aspects of the work.

\section{Funding}

This study was supported by grants from the Health Ministry of China, National 863 project (No. 2006AA02A406), 973 project (No. 2007CB512004), National Natural Science Foundation of China (No. 81100864) and a grant (No. 2019CFB730) from Science and Technology Plan Project of Hubei province, China.

\section{Disclosure}

The authors declare that they have no conflicts of interest.

\section{References}

1. Virani SS, Alonso A, Benjamin EJ, et al. Heart disease and stroke statistics-2020 update: a report from the American Heart Association. Circulation. 2020;141(9):e139-e596. doi:10.1161/CIR.00000000 00000757

2. Virani SS, Alonso A, Aparicio HJ, et al. Heart disease and stroke statistics-2021 update: a report from the American Heart Association. Circulation. 2021;143(8):e254-e743. doi:10.1161/CIR.000000 0000000950

3. Chao BH, Yan F, Hua Y, et al. Stroke prevention and control system in China: CSPPC-Stroke program. Int J Stroke. 2021;16(3):265-272. doi:10.1177/1747493020913557

4. Feigin VL, Nguyen G; Collaborators GBDLRoS. Global, regional, and country-specific lifetime risks of Stroke, 1990 and 2016. N Engl J Med. 2018;379:2429-2437.

5. Hecquet C, Tan F, Marcic BM, Erdos EG. Human Bradykinin B2 receptor is activated by kallikrein and other serine proteases. $\mathrm{Mol}$ Pharmacol. 2000;58(4):828-836. doi:10.1124/mol.58.4.828

6. Chao J, Bledsoe G, Yin H, Chao L. The tissue kallikrein-kinin system protects against cardiovascular and renal diseases and ischemic stroke independently of blood pressure reduction. Biol Chem. 2006;38 7:665-675.

7. Chao J, Chao L. Kallikrein-kinin in stroke, cardiovascular and renal disease. Exp Physiol. 2005;90(3):291-298. doi:10.1113/expphysi ol.2004.028464

8. Potier L, Waeckel L, Richer C, Roussel R, Bouby N, Alhenc-Gelas F. Tissue kallikrein, blood pressure regulation, and hypertension: insight from genetic kallikrein deficiency. Biol Chem. 2013;394(3):329-333. doi:10.1515/hsz-2012-0332

9. Feng J, Dong C, Long Y, et al. Elevated Kallikrein-binding protein in diabetes impairs wound healing through inducing macrophage M1 polarization. Cell Commun Signal. 2019;17(1):60. doi:10.1186/ s12964-019-0376-9

10. Chao J, Chao L. Experimental kallikrein gene therapy in hypertension, cardiovascular and renal diseases. Pharmacol Res. 1997;35 (6):517-522. doi:10.1006/phrs.1997.0179

11. Zhang Q, Ding H, Yan J, et al. Plasma tissue kallikrein level is negatively associated with incident and recurrent stroke: a multicenter case-control study in China. Ann Neurol. 2011;70 (2):265-273. doi:10.1002/ana.22404

12. Yang J, Su J, Wan F, et al. Tissue kallikrein protects against ischemic stroke by suppressing TLR4/NF-kappaB and activating Nrf2 signaling pathway in rats. Exp Ther Med. 2017;14(2):1163-1170. doi:10.3892/etm.2017.4614
13. Shi R, Yuan $\mathrm{K}, \mathrm{Hu} \mathrm{B}$, et al. Tissue kallikrein alleviates cerebral ischemia-reperfusion injury by activating the B2R-ERK1/2-CREBBcl-2 signaling pathway in diabetic rats. Oxid Med Cell Longev. 2016;2016:1843201. doi:10.1155/2016/1843201

14. Zhang Q, Ran X, Wang DW. Relation of plasma tissue kallikrein levels to presence and severity of coronary artery disease in a Chinese population. PLoS One. 2014;9(3):e91780. doi:10.1371/ journal.pone. 0091780

15. Li H, Pei F, Shao L, et al. Prevalence and risk factors of abnormal left ventricular geometrical patterns in untreated hypertensive patients. BMC Cardiovasc Disord. 2014;14(1):136. doi:10.1186/1471-2261$14-136$

16. Slee VN. The international classification of diseases: ninth revision (ICD-9). Ann Intern Med. 1978;88(3):424-426. doi:10.7326/00034819-88-3-424

17. Kushner FG, Hand M, Smith SC Jr, et al. 2009 focused updates: ACC/AHA guidelines for the management of patients with ST-elevation myocardial infarction (updating the 2004 Guideline and 2007 Focused Update) and ACC/AHA/SCAI Guidelines on Percutaneous Coronary Intervention (updating the 2005 guideline and 2007 focused update): a report of the American College of Cardiology Foundation/American Heart Association Task Force on Practice Guidelines. Circulation. 2009;120(22):2271-2306. doi:10.1161/CIRCULATIONAHA.109.192663

18. 1999 World Health Organization-International Society of Hypertension Guidelines for the Management of Hypertension. Guidelines Subcommittee. J Hypertens. 1999;17(2):151-183.

19. Alberti KG, Zimmet PZ. Definition, diagnosis and classification of diabetes mellitus and its complications. Part 1: diagnosis and classification of diabetes mellitus provisional report of a WHO consultation. Diabet Med. 1998;15(7):539-553. doi:10.1002/(SICI) 1096-9136(199807)15:7<539::AID-DIA668>3.0.CO;2-S

20. Brash AR. Arachidonic acid as a bioactive molecule. J Clin Invest. 2001;107(11):1339-1345. doi:10.1172/JCI13210

21. Ader JL, Tran-van T, Praddaude F. Renal tissue kallikrein in newborn and young SHR. Am $J$ Hypertens. 1988;1(3 Pt 3):53S-55S. doi:10.1093/ajh/1.3.53S

22. Wang J, Xiong W, Yang Z, et al. Human tissue kallikrein induces hypotension in transgenic mice. Hypertension. 1994;23(2):236-243. doi:10.1161/01.HYP.23.2.236

23. Yuan G, Deng J, Wang T, et al. Tissue kallikrein reverses insulin resistance and attenuates nephropathy in diabetic rats by activation of phosphatidylinositol 3-kinase/protein kinase B and adenosine 5'monophosphate-activated protein kinase signaling pathways. Endocrinology. 2007;148(5):2016-2026. doi:10.1210/en.2006-0602

24. Chao J, Yin H, Gao L, et al. Tissue kallikrein elicits cardioprotection by direct kinin $\mathrm{b} 2$ receptor activation independent of kinin formation. Hypertension. 2008;52(4):715-720. doi:10.1161/HYPERTENSION AHA.108.114587

25. Robles NR, Cerezo I, Hernandez-Gallego R. Renin-angiotensin system blocking drugs. J Cardiovasc Pharmacol Ther. 2014;19 (1):14-33. doi:10.1177/1074248413501018

26. Regoli D, Gobeil F. Kinins and peptide receptors. Biol Chem. 2016;397(4):297-304. doi:10.1515/hsz-2015-0240

27. Bader M. Molecular interactions of vasoactive systems in cardiovascular damage. J Cardiovasc Pharmacol. 2001;38(Suppl 2):S7-9. doi:10.1097/00005344-200111002-00003

28. Wang T, Hou LB, Liu ZJ, et al. Intramuscular delivery of rAAV-mediated kallikrein gene reduces hypertension and prevents cardiovascular injuries in model rats. Acta Pharmacol Sin. 2007;28 (12):1898-1906. doi:10.1111/j.1745-7254.2007.00677.x

29. Ribeiro MS, Dellalibera-Joviliano R, Becari C, et al. Characterization of the kallikrein-kinin system, metalloproteinases, and their tissue inhibitors in the in-stent restenosis after peripheral percutaneous angioplasty. Ann Vasc Surg. 2014;28(4):1005-1015. doi:10.1016/j. avsg.2013.11.014 
30. Tang M, He F, Ma L, Liu P, Wang J, Zhu X. Bradykinin receptors in ischemic injury. Curr Neurovasc Res. 2018;15(4):359-366. doi:10.2174/1567202616666181123151629

31. Murakami H, Yayama K, Miao RQ, Wang C, Chao L, Chao J. Kallikrein gene delivery inhibits vascular smooth muscle cell growth and neointima formation in the rat artery after balloon angioplasty. Hypertension. 1999;34(2):164-170. doi:10.1161/01.HYP.34.2.164

32. Chao J, Shen B, Gao L, Xia CF, Bledsoe G, Chao L. Tissue kallikrein in cardiovascular, cerebrovascular and renal diseases and skin wound healing. Biol Chem. 2010;391(4):345-355. doi:10.1515/bc.2010.042

33. Liu Y, Hashizume K, Chen Z, et al. Correlation between bradykinin-induced blood-tumor barrier permeability and B2 receptor expression in experimental brain tumors. Neurol Res. 2001;23 (4):379-387. doi:10.1179/016164101101198596

34. van Diepen JA, Berbee JF, Havekes LM, Rensen PC. Interactions between inflammation and lipid metabolism: relevance for efficacy of anti-inflammatory drugs in the treatment of atherosclerosis. Atherosclerosis. 2013;228(2):306-315. doi:10.1016/j.atheroscler osis.2013.02.028
35. Charla E, Mercer J, Maffia P, Nicklin SA. Extracellular vesicle signalling in atherosclerosis. Cell Signal. 2020;75:109751. doi:10.1016/j.cellsig.2020.109751

36. Montanari D, Yin H, Dobrzynski E, et al. Kallikrein gene delivery improves serum glucose and lipid profiles and cardiac function in streptozotocin-induced diabetic rats. Diabetes. 2005;54 (5):1573-1580. doi:10.2337/diabetes.54.5.1573

37. Wei Z, Lyu Y, Yang X, Chen X, Zhong P, Wu D. Therapeutic values of human urinary kallidinogenase on cerebrovascular diseases. Front Neurol. 2018;9:403. doi:10.3389/fneur.2018.00403

38. Ma N, Zhao ZA, Zhang NN, Chen HS. Intra-arterial human urinary kallidinogenase alleviates brain injury in rats with permanent middle cerebral artery occlusion through $\mathrm{PI} 3 \mathrm{~K} / \mathrm{AKT} / \mathrm{FoxO} 1$ signaling pathway. Brain Res. 2018;1687:129-136. doi:10.1016/j.brainres.20 18.02.049

39. Miao J, Deng F, Zhang Y, Xie HY, Feng JC. Exogenous human urinary kallidinogenase increases cerebral blood flow in patients with acute ischemic stroke. Neurosciences. 2016;21:126-130.
Journal of Inflammation Research

\section{Publish your work in this journal}

The Journal of Inflammation Research is an international, peerreviewed open-access journal that welcomes laboratory and clinical findings on the molecular basis, cell biology and pharmacology of inflammation including original research, reviews, symposium reports, hypothesis formation and commentaries on: acute/chronic inflammation; mediators of inflammation; cellular processes; molecular

\section{Dovepress}

mechanisms; pharmacology and novel anti-inflammatory drugs; clinical conditions involving inflammation. The manuscript management system is completely online and includes a very quick and fair peerreview system. Visit http://www.dovepress.com/testimonials.php to read real quotes from published authors. 